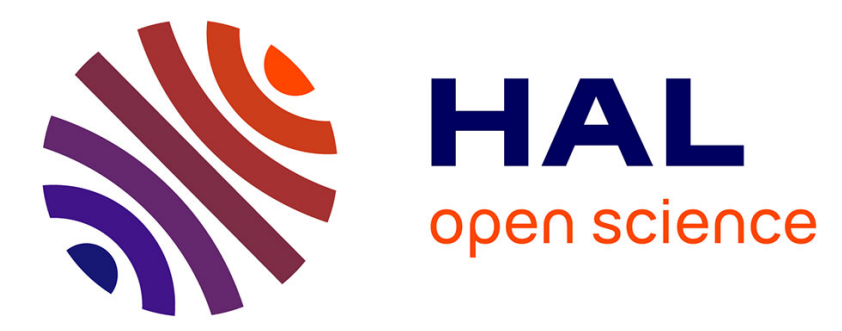

\title{
"Marchés transitionnels" du travail et restructurations : vers une gestion collective des transitions
}

Bernard Gazier

\section{To cite this version:}

Bernard Gazier. "Marchés transitionnels" du travail et restructurations : vers une gestion collective des transitions. La Revue de l'IRES, 2005, 47, pp.301 - 317. halshs-00270216

\section{HAL Id: halshs-00270216 \\ https://shs.hal.science/halshs-00270216}

Submitted on 4 Apr 2008

HAL is a multi-disciplinary open access archive for the deposit and dissemination of scientific research documents, whether they are published or not. The documents may come from teaching and research institutions in France or abroad, or from public or private research centers.
L'archive ouverte pluridisciplinaire HAL, est destinée au dépôt et à la diffusion de documents scientifiques de niveau recherche, publiés ou non, émanant des établissements d'enseignement et de recherche français ou étrangers, des laboratoires publics ou privés. 


\section{« MARCHES TRANSITIONNELS DU TRAVAIL » ET RESTRUCTURATIONS : VERS UNE GESTION COLLECTIVE DES TRANSITIONS}

Bernard Gazier

MATISSE

CNRS - U. Paris 1

Janvier 2005

\section{Introduction}

Cette contribution vise à présenter et discuter quelques apports d'un programme de recherche et d'action européen, les «marchés transitionnels du travail», face aux restructurations, aux licenciements collectifs et aux défis que pose le reclassement des travailleurs en France comme dans de nombreux pays. A première vue, ces apports sont de trois ordres.

Elaborée depuis 1995, la perspective européenne des «marchés transitionnels » propose tout d'abord une vision d'emblée dynamique du marché du travail et donc du déroulement des restructurations, et élabore des principes et critères visant à juger de la qualité des «transitions» auxquelles elles donnent lieu, compte tenu notamment des politiques publiques qui les accompagnent.

Ensuite, elle permet de se référer, dans ce cadre conceptuel commun, à une variété de pratiques locales et d'expériences nationales dont certaines, plus efficaces ou moins inefficaces que chez nous, sont susceptibles d'enrichir le débat français.

Enfin, elle conduit à replacer ces trajectoires plus ou moins contraintes et maîtrisées dans la dynamique d'ensemble des transitions qui se produisent dans l'emploi, sur le marché du travail ou autour de lui, pour déboucher sur une vision d'ensemble à la fois positive et normative.

Nous allons préciser ce point de vue, qui apparaît comme un élargissement systématique de compréhension et d'action, au delà de l'appréciation isolée d'une restructuration et sa prise en charge après coup. Il sera d'abord nécessaire d'évoquer les traits principaux des « marchés transitionnels du travail » (MTT désormais), pour évoquer quelques uns des débats auxquels ils ont donné lieu (section 1). On pourra ensuite appliquer les critères des « bonnes » transitions aux pratiques et difficultés actuelles, pour ouvrir sur une discussion plus générale (section 2). 


\section{LES MARCHES TRANSITIONNELS DU TRAVAIL : POINTS DE REPERE}

Les MTT ont fait l'objet de présentations relativement nombreuses (voir Schmid et Gazier (dir) (2002) ou Gazier (2003a) pour des exposés développés et Gazier (2003b) pour une synthèse récente), c'est pourquoi nous nous bornerons ici à un rappel succinct (1.1) avant d'approfondir quelques points de débat concernant l'orientation générale et les développements récents de cette approche (1.2)

\subsection{Un programme de recherche et d'action évolutif}

Issus d'une réflexion pragmatique menée au début des années 1990 par les économistes du Wissenschaftzentrum Berlin Günther Schmid et Peter Auer, les MTT ont constitué d'abord une perspective de réforme des politiques de l'emploi. Prenant acte du brouillage croissant des frontières entre travail rémunéré, activités personnelles et activités socialement utiles, leurs promoteurs se sont centrés sur les «transitions » qu'une personne peut accomplir au sein du marché du travail et autour de celui-ci, pour identifier les " transitions » souhaitables et leur associer de nouveaux droits. Au départ, le constat de l'échec de certaines politiques de l'emploi enfermant les chômeurs de longue durée dans des impasses ou des circuits de perdants, et l'effort pour mettre en oeuvre des politiques réintégratrices et préventives, élargissant le domaine d'action et associant une multiplicité d'acteurs locaux dans un cadre décentralisé. Les MTT ont été définis comme l'aménagement systématique et négocié des «transitions", ces dernières correspondant à l'ensemble des mobilités (changement d'emploi ou de statut, congés divers, promotions...). Systématique parce que les «transitions» sont vues dans leurs interactions ; négocié parce que les politiques publiques de l'emploi sont trop souvent unilatérales et doivent être réinsérées dans la gestion, par les acteurs eux-mêmes, de leurs trajectoires professionnelles et personnelles.

Pratiquement, il s'agit de lutter contre l'exclusion en multipliant les opportunités disponibles pour les travailleurs, et en mettant en quelque sorte sous pression les entreprises. Les politiques actives de l'emploi telles qu'elles ont été développées au Danemark constituent un exemple, parmi d'autres, d'une telle approche.

Toutefois cette première visée, déjà ambitieuse, a débouché, au tournant du siècle, sur un second élargissement, plus considérable encore, du champ d'analyse et d'action. En effet, une objection a été rapidement formulée à l'encontre de cette démarche: faute d'agir directement sur les entreprises, on risque d'aboutir à un cercle vicieux. Les travailleurs pris en charge par les congés ou les politiques de l'emploi risquent en effet de n'être que temporairement ré - intégrés par les entreprises, qui pourraient se contenter de les utiliser dans des postes précaires et les renvoyer rapidement aux "politiques actives de l'emploi ». Les responsables publics auraient donc de nouveau à placer/former/motiver ces mêmes travailleurs pour les renvoyer aux entreprises, etc. Par un effet «tonneau des Danaïdes », les MTT faciliteraient des comportements court - termistes des entreprises, en se bornant à socialiser leurs conséquences. 
Il faut toutefois constater que les «transitions » ont d'emblée concerné les carrières des salariés au sein des entreprises tout autant que leurs mouvements hors des entreprises : le passage du temps complet au temps partiel (et vice et versa), la modulation des horaires, le régime des promotions, sont autant d'éléments à prendre en compte qui interdisent de se limiter aux politiques publiques de l'emploi menées hors des entreprises. C'est pourquoi une seconde perspective de recherche et d'action est apparue dès le tournant du siècle (voir Gazier et Schmid (2001), qui prône une réforme de la relation salariale modifiant la répartition des risques et des opportunités découlant des activités productives. En particulier, les MTT préconisent un accroissement des droits et des options à la disposition des salariés, leur permettant de rééquilibrer un partage du pouvoir indûment déséquilibré en faveur des actionnaires.

Si l'on considère ensemble les propositions de réforme tant des politiques publiques de l'emploi que de la relation salariale, un troisième élargissement se dessine, qui ne sera que mentionné ici : les MTT peuvent constituer le noyau central d'un nouveau modèle social, alternatif au modèle social libéral tel qu'il a été par exemple élaboré par A. Giddens (1998). A grands traits, ce modèle «social - démocrate renouvelé » cherche non seulement à " équiper les gens pour le marché », mot d'ordre caractéristique du social - libéralisme, mais aussi à " équiper le marché pour les gens » (voir Gautié et Gazier (2003)). En effet, il s'agit de compléter la protection sociale classique fournie par l' Etat - Providence traditionnel, par de nouveaux droits, sous la forme de Droits de Tirage Sociaux, dont la mise en œuvre est contrôlée collectivement.

Cette dynamique de recherche une fois posée, on peut revenir brièvement sur quelques traits distinctifs des MTT.

Les principaux champs des « transitions » sont bien connus : au sein de l'emploi, entre emploi et formation, entre emploi et inactivité (retraite, handicap), entre emploi et chômage, entre emploi et activités sociales utiles (activités domestiques, bénévoles, militantes).

Quatre principes permettent alors de définir les "bonnes transitions".

(1) Le premier principe est d'accroître la liberté individuelle (ou l'autonomie), en donnant aux personnes plus de pouvoir, non seulement en termes financier via des transferts mais aussi en termes de participation aux décisions d'emploi qui les concernent. En échange de quoi les participants au marché du travail pourront accepter d'assumer plus de risques, plus de devoirs et d'obligations.

(2) Un second principe est de promouvoir la solidarité dans la gestion des risques sociaux et des risques associés au marché du travail. Ceci implique l'inclusion, dans les programmes de redistribution, des travailleurs les plus favorisés, qui courent moins de risques ou sont mieux à même de les assurer.

(3) Le troisième principe consiste à rechercher l'efficacité des mesures accompagnant les transitions, à travers un processus de spécialisation, coordination et coopération. Celui-ci prend le plus souvent la forme d'un mélange de contributions publiques et privées dans la formulation et la mise en œuvre des politiques correspondantes, et suppose des modalités négociées de prise de décision. 
(4) Enfin, le dernier principe consiste à mobiliser l'arsenal des techniques de gestion des risques : contrôle, évaluation et auto-régulation, par le biais d'une démarche largement décentralisée ou par le management par objectifs. Ce principe requiert la recherche d'une meilleure codétermination des acteurs concernés, que ce soit au niveau de la firme, au niveau local ou au niveau régional.

Parmi les autres traits des MTT qui doivent être mentionnés, il convient de signaler l'arrivée de nouveaux acteurs et financeurs dans les négociations autour de l'emploi et des transitions. Par exemple, organiser des préretraites à temps partiel $^{1}$ peut se faire avec l'organisation de compléments d'emploi du temps au sein d'associations qui n'ont pas les moyens de financer un emploi à temps plein et peuvent accueillir des collaborateurs à temps partiel - cet emploi pouvant du reste être co-financé par un partenaire municipal ou régional. Un tel dispositif a l'intérêt de compléter les revenus et l'activité des travailleurs vieillissants, en satisfaisant des besoins sociaux et sans alourdir indûment la charge des finances publiques.

On l'a dit, la démarche des MTT est une démarche d'ensemble : ma mobilité dépend de la vôtre. Il en résulte l'insistance mise sur des négociations locales associant de multiples acteurs susceptibles d'être co-financeurs. Une série de questions de mise en œuvre surgit alors, portant notamment sur le coût et la cohérence de ces arrangements locaux. En ce qui concerne le coût, les MTT consistent d'abord en réaménagement des mesures existantes, et prennent appui sur les avantages des co-financements y compris en provenance du travailleur bénéficiaire de la «transition », situations dans lesquelles chaque participant a intérêt au succès de la «transition». Plus que des dépenses nouvelles, il s'agit plutôt de la réappropriation par les acteurs locaux des sommes souvent très importantes consacrées par l'Etat aux politiques de l'emploi. On peut donc établir des conditions de maîtrise des coûts. La question de la cohérence apparaît par exemple lorsque l'on envisage des régions riches qui pourraient proposer un ensemble sophistiqué de «transitions» pendant que des régions pauvres en sont réduites à une offre minimale. Le moyen de combattre cette incohérence est une politique de transferts assurée par l'Etat-nation (ou l'Europe), qui suppose d'élaborer et d'utiliser des critères de besoins locaux en termes de « transitions ».

\subsection{Quelques questions d'interprétation}

Les MTT ont suscité de nombreux débats et objections qui sont autant d'occasion de clarifier une problématique dont on vient de souligner la plasticité et la dynamique expansive. Nous en évoquerons brièvement cinq.

- 1. l'éloge indifférencié de la mobilité ?

Par l'accent qu'ils mettent sur les «transitions », les MTT semblent faire la promotion d'un type de travailleur mobile et l'éloge de la mobilité pour elle-même. Certains ont ironisé sur les « travailleurs - voyageurs » que cette perspective amènerait à multiplier. On peut plus sérieusement craindre que les MTT ne conduisent à entériner les licenciements économiques

\footnotetext{
${ }^{1}$ La préretraite à temps plein n'est pas une mesure obéissant à la logique des MTT, sauf dans certains cas "sociaux". En effet elle est le plus souvent, quelque soit l'engouement dont elle bénéficie, irréversible, brutale, contraignante, unilatérale et coûteuse.
} 
ou autres, quels qu'ils soient, sous le prétexte de les anticiper ou de les accompagner. Cette critique découle de la focalisation initiale. Face à l'érosion des protections traditionnelles des travailleurs, au sein notamment des "marchés internes » et des carrières aménagées et sécurisées qu'ils organisaient, le parti-pris des MTT est d'élargir la base de leur sécurisation en faisant des parcours de mobilité le principal levier collectif d'intervention.

Les premières formulations des MTT, qui se centrent sur la réintégration des chômeurs de longue durée et insistent sur les actions à l'extérieur des entreprises ou en partenariat avec celles-ci, ont pu prêter le flanc à une telle interprétation. Toutefois l'élargissement à la réforme de la relation salariale lève l'équivoque. La démarche admet que les firmes puissent revendiquer adaptation et flexibilité, par exemple sous la forme de modulations d'horaires. Mais elle entend leur faire payer le prix social de celles-ci, par exemple sous la forme de comptes épargne - temps équilibrés ouvrant plus de choix aux travailleurs. D'autre part, le point de vue des MTT est que les entreprises ont à la fois besoin de stabilité et de flexibilité, tout comme les travailleurs. Il s' agirait donc, loin d'une valorisation générale et indistincte de toute mobilité, de définir une nouvelle norme d'emploi durable, en révélant ces besoins et en organisant leur confrontation équilibrée.

\section{- 2. l'éloge des travailleurs high-tech?}

La référence faite aux « Sublimes » du XIXe siècle français (Gazier (2003a)) a suscité un débat analogue. Faut-il faire l'éloge tous azimuts du comportement de ces ancêtres ultramobiles du mouvement ouvrier, qui prétendaient choisir leurs patrons et ne travaillaient que le temps de gagner un surplus destiné à être dépensé dans des activités de loisir ? et faut-il voir dans leurs lointains héritiers les travailleurs high-tech par projets de l'informatique, la préfiguration d'un statut ou de comportements à généraliser? Il convient tout d'abord de le pas mélanger les deux types de trajectoires. Les «Sublimes» mettaient en œuvre un hédonisme qui paraît bien loin des actuels travailleurs high-tech, pris dans une spirale compétitive sans pitié ni repos. La bonne référence est donc celle du passé. Mais l'essentiel de la leçon des "Sublimes » est dans le contrôle du marché du travail qu'ils avaient réussi à instaurer. Au lieu de s'accrocher à des garanties juridiques d'emploi, c'est par leur forte qualification et par leur emprise sur le marché du travail qu'ils ont pu, un temps et pour un petit groupe, atteindre ce mode de vie libertaire.

C'est cette leçon que les MTT entendent mettre en application, notamment par la mise en cause des compétitions excessives entre travailleurs, ce que l'on nomme parfois les « courses de rats », et par la promotion des activité sociales utiles au cours d'une carrière. Il ne s'agit donc pas simplement d'affronter les défis que ce que Boltanski et Chiapello (1999) ont nommé la « cité par projets », même si la vision dynamique des carrières personnelles et professionnelles est au cœur des deux problématiques.

\section{- 3. l'oubli de la segmentation débouchant sur son renforcement?}

On peut ici reprendre pour la systématiser l'objection reprochant aux MTT leurs voies d'action privilégiant l'organisation sociale des mobilités en dehors des entreprises. Non seulement les entreprises pourraient prendre appui sur les MTT pour mettre en œuvre une politique d'usage à court terme de leur main-d'œuvre, mais elles pourraient renforcer un processus plus général de tri et de segmentation. Par un jeu de vases communicants, l'instabilité socialisée des uns viendrait renforcer la mise sous pression des plus qualifiés et des plus favorisés. Cette objection appelle alors le même type de réponse. Comme on l'a vu, par la double réforme des politiques de l'emploi et de la relation salariale, c'est au contraire 
un processus de « dé - segmentation » si l'on peut dire qu'il s'agirait de lancer, en accordant davantage de droits tant aux « insiders » qu'aux « outsiders » et notamment aux plus faibles d'entre eux, et en mettant les entreprises sous la pression de possibilités de choix accrues de la part des travailleurs. Les bases nouvelles de stabilisation des travailleurs devraient s'appuyer à terme sur une nouvelle structuration des carrières, sans doute des "marchés internes" reconfigurés et organisés en réseaux. Cette nouvelle structuration n'a fait l'objet jusqu'à présent que de spéculations.

- 4. l'oubli de la dimension « genre » débouchant sur le renforcement des inégalités entre hommes et femmes?

L'observation des transitions existant actuellement, en se limitant aux mouvements entre l'entreprise et les champs de la formation, du chômage, de l'inactivité et des activités sociales utiles, fait apparaître que $60 \%$ des transitions sont effectuées par des femmes. Il en résulte une interrogation naturelle : mieux organiser ou simplement faciliter les transitions, au lieu de simplement développer l'accès à l'emploi « normal » et de sécuriser celui-ci, ne seraitce pas renforcer l'inégalité de «genre»? On remarquera d'abord que les MTT ont pour premier effet de rendre visible ce problème et d'en systématiser l'expression, ce qui est en soi une bonne chose. Ensuite, organiser les transitions dans la ligne des MTT signifie entre autres les rééquilibrer entre hommes et femmes, notamment en tenant compte de la répartition existante et socialement souhaitée des tâches domestiques. Un bon exemple de dispositif illustrant ces préoccupations et ces priorités est le congé parental suédois, quoi accorde la même importante durée aux deux parents pris isolément, et accorde une durée totale nettement plus importante aux deux conjoints s'ils font le choix de prendre l'un et l'autre la durée maximale (simultanément ou successivement). Il est clair que cette politique qui améliore l'égalité de "genre » ne peut fonctionner qu'avec un relais significatif au sein des firmes elles-mêmes. Il faut en effet supprimer ou du moins mitiger la "course de rats » évoquée ci-dessus, de manière à ce que chaque conjoint ait le même avantage (ou désavantage) à prendre un congé et à participer aux activités domestiques. Ce qui met en jeu les MTT comme réforme de la relation de travail tout autant que comme aménagement des mobilités.

- 5. l'éloge sans précautions du marché et de la flexibilité ?

On a pu enfin s'interroger sur l'intitulé même de "marché » servant d'emblème à l'approche et aux réformes envisagées. En des temps d'expansion envahissante de la logique marchande, n'est-il pas mal venu de prétendre développer ou même instaurer des « marchés »? L'éloge fait dans des milieux libéraux de la "flexicurité » hollandaise ou danoise ne vient-il pas ajouter une source supplémentaire de confusion en suggérant une lecture libérale de l'organisation de la flexibilité ?

Observons tout d'abord que le terme initial ne faisait pas cette référence, puisque G. Schmid et P. Auer parlaient d'instaurer des «passerelles » vers l'emploi normal. Ensuite, il s'agit bien par certains aspects de marchés, c'est-à-dire de processus socialement organisés de révélations et confrontations de besoins, et de cadres autorisant des transactions: sur les transitions. Le terme de "marché » se justifie dans la mesure exacte où l'on a pu parler de « marchés internes ». Cette référence antérieure au marché n'avait pas, tant s'en faut, la connotation précarisante que l'on constate de nos jours et désignait un processus intra-firme socialisé d'allocation de positions de travail et de rémunérations. Il s'agit désormais de processus intra et extra - firme socialisés d'allocation de positions d'activité et de rémunérations conçues comme étapes dans une carrière. 
Autrement dit, loin de promouvoir un libre de jeu de marché sans contrôle, les réformes inspirées par les MTT recherchent le ré - encastrement des mobilités sur le marché du travail, en rééquilibrant le pouvoir des acteurs au profit des moins puissants et en instaurant des connections explicitées et organisées avec le réseau des activités non marchandes de toute nature

Quant à l'éloge de la flexicurité désormais fait par bien des analystes, y compris l'OCDE (après des années passées à la promotion de la seule flexibilité), qu'il nous soit permis d'y voir l'heureuse banalisation d'une thématique plus équilibrée que la précédente. Les relectures « néolibérales » de la flexicurité montrent simplement qu'elle est à la mode, ce qui ne suffit pas pour la juger sans pertinence. Une perspective cousine, qui a l'avantage d'éviter les équivoques du terme de marché, est désormais celle que développent les chercheurs du BIT autour de Peter Auer : celle de la " mobilité protégée » (BIT (2004)). S'il paraît difficile de renoncer à l'étiquette désormais admise, c'est dans l'esprit de cette dernière dénomination que l'on peut situer les développements qui vont suivre, illustrant et discutant une lecture « transitionnelle » des restructurations.

\section{RESTRUCTURATIONS : QUELLES TRANSITIONS ?}

On commencera par présenter une opposition simple et délibérément tranchée entre un ensemble de pratiques peu satisfaisantes souvent constatées - et critiquées - dans notre pays en dépit des efforts qui y sont accomplis, et une démarche nationale bien affermie qui s'affranchit pour une part de ces difficultés : les «fondations de travail » en Autriche (2.1). Il sera alors possible de généraliser quelque peu le propos (2.2).

\subsection{Deux scénarii de reclassement}

On peut décomposer en cinq éléments le « scénario catastrophe » qui trop souvent se joue en France à l'occasion d'un plan social.

- 1. L'annonce aux syndicats et au public, différée au maximum, est brusque et instaure un climat d'urgence et d'angoisse. Le chiffrage des effectifs concernés et les modalités proposées de séparation sont tenues secrets le plus longtemps possible avant d'être révélés en bloc.

- 2. La procédure des «plans sociaux » est lancée, elle est complexe et lente. Une antenne de reclassement est instaurée.

- 3. Le choix des «partants » est effectué par l'entreprise ou un cabinet conseil qui sélectionne les moins adaptables, les moins qualifiés et les plus âgés pouvant bénéficier d'une mesure d'âge, au cours d'entretiens « à la chaîne ». Ce processus génère un climat social exécrable où les " partants » se sentent condamnés cependant que les « restants » se sentent en sursis et rasent les murs. Avant même d'avoir quitté l'entreprise, les «partants » ont mauvaise réputation, ils sont stigmatisés comme les « perdants ».

- 4. Les protestations des «partants», groupe souvent composé de salariés à forte ancienneté et forte syndicalisation, leur permettent d'obtenir l'appui des pouvoirs publics locaux et nationaux. Outre l'aide au reclassement initialement prévue, ils obtiennent d'autres ressources, parfois massives mais ponctuelles. Les actions de 
formation demeurent limitées, compte tenu notamment de l'âge moyen des personnes concernées.

- 5. Il en résulte de fortes difficultés de reclassement. Typiquement, au bout de deux ans, la moitié des « partants » ont retrouvé un emploi.

On observe ici l'enchaînement de deux injustices. La première est celle qui consiste à isoler des « perdants » pour les stigmatiser. La seconde consiste à surcompenser la première en accordant au groupe des «partants», des aides supplémentaires massives auxquelles les autres chômeurs, n'ayant pas été pris dans le processus de licenciement collectif, n'auront pas droit.

Ce scénario noir de la déchirure et de l'impasse peut ainsi se produire en dépit d'efforts intenses accomplis par les pouvoirs publics et les collectivités locales, par les " partants » eux-mêmes voire par les firmes. Parmi les points d'appui offerts au nom de la solidarité, on doit mentionner le recours systématique aux préretraites totales, censées fournir une porte de sortie honorable à des «seniors » jugés peu adaptables et excédentaires, et les tentatives, au moins verbales, de s'engager dans une gestion prévisionnelle de l'emploi et des compétences, censée permettre d'éviter dans le futur les situations de rejet massif des salariés. On sait toutefois que les préretraites ne se justifient que dans des cas bien précis qui en font une mesure « sociale » destinée à certaines catégories de travailleurs prématurément " usés », et sont porteuses d'inconvénients multiples. Et que la «gestion prévisionnelle » n'a guère dépassé le stade des vœux pieux.

L'expérience autrichienne des « Fondations de travail » Voest - Alpine Stahlstifstung date de 1987 et remonte aux initiatives prises par les partenaires sociaux à l'occasion de licenciements effectués par la grande entreprise sidérurgique Voest - Alpine. Elle a été attentivement étudiée et évaluée (Winter - Ebmer (2001)). Reprenons terme à terme les éléments du scénario précédent.

- 1. L'annonce de la restructuration est faite au moins six mois à l'avance, de manière à laisser de la place aux concertations collectives et aux initiatives individuelles

- 2. L'antenne de reclassement est une fondation, ce qui veut dire qu'elle repose sur un capital immobilisé pour elle. Elle est donc indépendante et stable, et subsistera autant que de besoin. Outre son capital, elle bénéficie de trois apports de ressources : des fonds publics des politiques de l'emploi, une partie significative de l'indemnité de reclassement touchée par les "partants », et une très faible cotisation payée par les salariés qui restent (de l'ordre de $0,2 \%$ de la masse salariale), destinée à payer les actions de formation.

- 3. Dans ce contexte, les «partants » sont des volontaires, qui peuvent être d'âge et de qualification très variés (reflétant la composition moyenne de l'établissement concerné), et sont identifiés comme des salariés dynamiques saisissant une occasion de progresser dans leur carrière ou de diversifier leur expérience. Il en résulte que le climat social est bien meilleur, et que notamment la solidarité est maintenue entre les « partants » et les « restants ».

- 4. Le traitement des "partants» se fait en privilégiant les actions de formation, qui sont perçues comme une composante centrale de la démarche de reclassement.

- $\quad 5$. Il en résulte des performances de reclassement qui sont supérieures.

Il importe de ne pas « rosir » à l'excès ce second scénario. Rien dans ce qu'il articule ne crée des emplois. En revanche il ne détruit pas l'employabilité des travailleurs en 
reconversion, et mieux, il la renforce. On peut facilement montrer que, même pris isolément et avec cette limite, il satisfait les quatre principes de gestion des " marchés transitionnels » présentés dans notre section 1 . Tout d'abord il instaure plus de choix pour les salariés, et leur donne un certain pouvoir de choisir («empowerment»). Ensuite il construit la solidarité entre les différentes catégories de travailleurs, ici les « partants » et les « restants ». La recherche de l'efficacité est elle aussi visible : tout le monde a intérêt à ce que le reclassement se termine vite et bien. L'entreprise, qui pourra à terme récupérer son capital, les « restants » qui payent une cotisation, les " partants » eux-mêmes qui abandonnent une part de leurs indemnités dans un processus de co-investissement. Enfin, la gestion du processus est décentralisée, et la Voest - Alpine Stahlstifstung a servi d'organisation - ombrelle pour accueillir les antennes de reclassement d'autres entreprises

S'agit-il simplement de « bonnes pratiques » pas reproductibles hors de leur contexte ? et comment dépasser les limites d'une solidarité locale si bien construite soit-elle?

\subsection{Gestion élargie des reclassements et implication des parties prenantes}

Poussons tout de suite le curseur à son terme. On est doublement fondé à vouloir radicalement dépasser voire minorer le point de vue circonscrit à la gestion, même anticipée et négociée, des restructurations. D'une part de nombreux travaux (dont ceux de R. Beaujolin 1998) ont mis en évidence à quel point l'identification et la gestion des sureffectifs sont des construits sociaux, dépendant de conventions elles-mêmes arbitraires et notamment répercutant la domination actuelle de la sphère financière sur les choix « réels ». D'autre part, en amont et en quelque sorte au dessus des processus en cause, on trouve bien évidemment les choix stratégiques effectués par les firmes (choix des produits, des techniques, de la localisation des investissements...) et le cadre macro-économique dans lequel ils sont effectuées (croissance, taux de change, fiscalité...). Nous voudrions ici plaider pour l'utilité d'un point de vue intermédiaire : celui des transitions, qui, sans récuser l'importance de ces construits et de ces déterminants, reste centré sur le terrain des ajustements de l'emploi et des trajectoires. En effet deux risques symétriques apparaissent ici. Le premier est de se désintéresser des trajectoires et des conditions de leur gestion, au motif que, pour partie (mais jusqu'à quel point ?) le diagnostic et l'action doivent se situer en amont ou au dessus. Symétriquement, s'en tenir aux mouvements affichés de l'emploi conduit à ignorer les dimensions de construit social et les déterminants stratégiques et macroéconomiques, ce qui est susceptible de contraindre indûment les options auxquelles doivent se résigner les acteurs concernés.

Nous l'avons suggéré dans notre première section : le double élargissement des MTT est susceptible d'apporter des éléments de réponse à ce défi intellectuel et pratique. Les «transitions » incluant le déroulement de la relation de travail, il ne s'agit pas d'une vision d'équilibre partiel élargi à l'ensemble du marché du travail, mais d'une vision moyenne ouverte sur l'ensemble des relations entre capital et travail. Le défi est d'assurer une complémentarité entre des politiques de croissance et de création d'activités, et l'action sur les « transitions », qui elle même doit pouvoir les faciliter.

La question centrale que l'on isole alors est celle de la responsabilité et de l'implication que l'on peut ou doit demander aux différentes parties prenantes dans le domaine du reclassement. Elle se pose d'abord en ce qui concerne les firmes qui licencient et 
dans le cas d'une réponse affirmative, il faut pouvoir préciser le degré et des modalités d'expression de cette responsabilité. Le débat dans ce domaine est aujourd'hui particulièrement vif en France. En témoignent les propositions radicales faites à l'automne 2004 par deux économistes recommandant de rendre possible le désengagement total des firmes en échange du versement d'une «contribution de solidarité » (Cahuc et Kramarz (2004)). Avec d'autres, ces économistes ont fait rentrer dans le débat français le thème de la « flexicurité » et l'éloge de l'expérience du Danemark dans le but essentiel de mettre en cause le droit existant du licenciement collectif, dont ils soulignent le caractère procédurier et contraignant. Il n'y a pas lieu dans le cadre de cette contribution, d'examiner plus en détail le contenu de ce rapport ${ }^{2}$. Il envisage la marginalisation des dispositifs et aides spécifiques en faveur des travailleurs subissant des restructurations (quelques entreprises volontaires pourront effectuer des démarches de reclassement et ne pas payer la «contribution de solidarité »). Ces travailleurs sont purement et simplement renvoyés à un traitement d'ensemble du chômage: pressions en faveur de l'intensification des efforts de recherche accomplis par les chômeurs, et politique visant à proposer et faire accepter principalement des postes de travail faiblement qualifiés. Sortie «par le bas » en quelque sorte, qui approfondit la tendance française à la « flexibilité administrée » ${ }^{3}$.

On doit commencer par observer que le contexte dans lequel s'est instaurée l'expérience danoise est tout à fait spécifique et aux antipodes du cas français : tissu productif fait très majoritairement de PME, taux de syndicalisation record $(80 \%)$, longue tradition de dialogue social et confiance réciproque entre les partenaires sociaux. L'activisme public est ainsi un choix qui fait en définitive de l'Etat danois un employeur en dernier ressort mais aussi voire davantage un investisseur social favorisant la formation et l'adaptation des salariés, ce qui induit une sortie «par le haut» des difficultés de reclassement.

Ensuite, ce qui est nécessaire ici est la contextualisation du cas français lui-même, de ses difficultés comme de ses marges de manœuvre. Sans entrer dans des détails qui nous écarteraient de l'axe central de notre sujet, on doit évoquer le processus assez particulier de constitution des "marchés internes » dans notre pays, et la liaison particulièrement faible voire déficiente entre notre appareil éducatif et notre appareil productif. Face à une longue tradition de coupure entre travail manuel et intellectuel, à une gestion frileuse d'un système éducatif général dont les élites ont cherché longtemps davantage le contrôle que la professionnalisation, le comportement des grandes firmes a été double : d'une part pousser le plus loin possible la taylorisation, et d'autre part opérer une compensation partielle de ces défaillances éducatives par l'instauration de carrières aménagées destinées à des travailleurs initialement peu qualifiés et formés sur le tas. Il en est résulté, durant les années 1950 et 1960, le développement de "marchés internes » qui sont rapidement apparus comme limités et rigides. Ce détour permet de comprendre l'axe dominant des comportements actuels, identifié il y a plus de vingt ans par J. Freyssinet (Freyssinet (1982)) : réduction et flexibilisation du «noyau dur» antérieurement constitué par sélection d'une main-d'œuvre qualifiée et

\footnotetext{
${ }^{2}$ Ils entendent opérer un déplacement de responsabilité et d'action : des procédures d'indemnisation brèves mais plus généreuses ont pour contrepartie un contrôle renforcé et centralisé des efforts de recherche d'emploi, cependant que des mesures d'ouverture à la concurrence, surtout sur le plan des marchés des produits (notamment dans le commerce) et plus accessoirement sur celui du marché du travail (pour des professions telles que chauffeur de taxi, vétérinaire, coiffeur) visent à multiplier des opportunités d'emplois. Cette démarche se centre sur un jeu de contreparties et de rééquilibrages dont le cœur repose sur un diagnostic simple : la plus grande partie des chômeurs français subissent cette situation parce que leur productivité est inférieure au niveau de rémunération garanti par le salaire minimum, ou trop proche de celui-ci (voir Cahuc et Zylberberg (2004)). ${ }^{3}$ Ces propositions re - centralisent paradoxalement les politiques de l'emploi, en une époque où la plupart des acteurs et des observateurs insistent sur leur nécessaire décentralisation.
} 
adaptable, et rejet des salariés antérieurement stabilisés dont le potentiel d'adaptation apparaît insuffisant.

De ce point de vue, les propositions de désengagement sélectif des grandes firmes en France constituent une tendance ancienne, avivée par l'arsenal juridique réglementant le droit du licenciement collectif. Dans le contexte français et sans les contreparties et contrepoids danois, on peut $s$ 'attendre à un effet destructeur massif.

Quelle serait une gamme d'actions et de politiques permettant d'organiser l'implication des entreprises et d'autres acteurs, locaux et nationaux, dans une démarche qui, sans crispation réglementaire, valoriserait l'existant pour aller vers une "sortie par le haut»? Des démarches de restructurations anticipées et responsables ont certes été observées en France : certaines grandes entreprises, notamment Saint-Gobain, fortes il est vrai d'une maind'œuvre de plus en plus qualifiée, ont pu gérer de manière concertée des reconversions en générant un tissu productif de substitution par des partenariats soutenant la création d'activités nouvelles (Raveyre 2001). Au delà de ces exemples isolés, bien des efforts ont été déployés en France pour «mobiliser les acteurs », « revitaliser l'emploi local» dans des bassins sinistrés, et attirer de nouvelles activités - avec un succès mitigé. Ce qu'apporte dans ce cadre la perspective des MTT est double.

D'abord, l'insistance sur les conditions de l'implication efficiente des différents acteurs, y compris les entreprises qui licencient, dans un cadre de négociations locales mobilisant un espace élargi de transitions. On peut sûrement penser à instaurer des " Fondations de travail » à l'autrichienne pour les entreprises qui en ont les moyens et qui réduisent leurs effectifs sans mette la clé sous la porte. Mais l'implication des firmes, de toutes tailles, sera d'autant meilleure qu'elles pourront anticiper leur adaptation et compter sur des outils publics préservant l'employabilité. On sait par exemple qu'en France les préretraites à mi-temps (ou progressives) ont été un échec. Peu incitatives, elles semblaient empêcher les entreprises de faire place nette. Elles étaient perçues par les travailleurs « seniors » vulnérables comme une demi - réalisation du droit acquis auquel ils aspiraient : se retirer plus tôt pour passer à l'abri d'une concurrence de plus en plus dure. Pour être efficaces, elles supposent en effet une concertation, et le chiffrage puis la prise en charge négociée de certains coûts. Garder des salariés à mi-temps nécessite d'actualiser leur formation, d'organiser des plannings et parfois de dupliquer des bureaux ou des postes, ce qui est coûteux pour l'entreprise et doit être financé par la collectivité. Organiser des mi-temps complémentaires, par exemple dans un réseau d'associations, demande un savoir-faire et un effort de coordination comme savent le réaliser les Groupements d'Employeurs, avec là aussi la nécessité d'investissements dans une formation professionnelle spécifique. La démultiplication des marges de manœuvre ainsi obtenue permet de faire apparaître davantage d'opportunités pour les travailleurs en reconversion. On retrouve ici le gisement d'emplois du tertiaire, dans un éventail plus vaste d'activités relationnelles et sociales, et avec un éventail de rémunérations collectivement agréé

Une telle démarche fait le pari de rechercher de nouvelles complémentarités ou synergies, cherchant une " destruction créatrice » non seulement socialement contrôlée, mais plus rentable économiquement en en faisant l'occasion d'un rebond. Elle est organisée localement, de manière à rendre gérable la complexité des transitions. Elle adosse la préservation des savoirs-faire existants à l'acquisition ou la révélation d'autres aptitudes professionnelles ou relationnelles. Elle cherche ainsi à concilier sécurité et mobilité, cependant que les entreprises qui licencient sont incitées à favoriser les démarches de transmission de leurs savoirs-faire, ce qui doit les conduire à une perspective de renouvellement progressif de leurs compétences. 
En effet, le second apport des MTT est de préciser les conditions d'une démarche préventive crédible. On a évoqué ci-dessus les difficultés voire les impasses de la GPEC traditionnelle, qui tentait d'allonger l'horizon de gestion des salariés dans un contexte marqué par un raccourcissement des prévisions et une exigence accrue d'adaptabilité. C'est seulement en articulant mobilité et fidélisation, dans une perspective transversale, que l'on peut restaurer une vision de long terme (Burzlaff et La Padellec (2001)). Ce qui implique la conjonction d'au moins trois éléments : une veille et des négociations sur l'employabilité, une politique de formation axée sur la valorisation de compétences transférables à toutes les étapes de la carrière et un partage intergénérationnel des risques associés au travail.

Les négociations sur l'employabilité restent en France quelque peu tabou, sans doute parce que le terme même d'employabilité semble ne renvoyer qu'à la responsabilité individuelle. Ce n'est en fait pas le cas (Gazier (2003c)). L'évolution récente des négociations collectives en Hollande est ici intéressante à retracer. L'accord signé en 1998 par NS/Dutch Railways prévoit que tous les trois ans la firme paye à chacun de ses 14000 salariés un bilan personnalisé de compétences et de carrière par un organisme indépendant. Si une actions de formation ou de recyclage apparaît nécessaire, l'employeur est tenu de la financer et de l'organiser, et le salarié est tenu de la suivre. Il y a ainsi une gestion concertée de droits et de devoirs réciproques. Cet accord cherche ainsi, non à garantir l'emploi antérieur, mais à garantir une maîtrise de carrière et des perspectives crédibles de ré - emploi pour les salariés.

La conséquence de l'érosion des "marchés internes » en France se lit aisément dans les politiques de formation des firmes qui, même lorsqu'elles sont développées, excluent les plus de 45 ans, ce qui en fait par la suite des victimes toutes désignées pour des préretraites. S'il est peu réaliste de prôner des dépenses massives de formation pour des salariés de plus de 55 ans, on peut en revanche suggérer de focaliser les efforts sur la tranche $45-55$, et de lier systématiquement chômage partiel et mesures de formation. Les multiples démarches de Validation des Acquis de l'Expérience vont elles aussi dans ce sens et peuvent recevoir un appui supplémentaire par une formation complémentaire apportant l'actualisation et renforçant la transférabilité des compétences.

L'idée de partage intergénérationnel des risques consiste à systématiser la pratique d'attribution à certains travailleurs de postes « doux », moins exigeants en réactivité physique ou nerveuse. Sans entrer ici dans le débat sur les liens entre âge, habileté et productivité, on se bornera à observer qu'il est naturel de diversifier les engagements productifs au cours du temps, en fonction des aptitudes et des disponibilités de chacun. Ceci doit correspondre à des rotations en ce qui concerne les postes les plus exigeants, et aussi à des horaires hebdomadaires modulables en fonction de l'âge et des desiderata personnels.

Pris ensemble, ces trois éléments pourront contribuer à une redéfinition des anciens « marchés internes », qui élargit et dynamise la base des garanties antérieurement consenties (pour une argumentation en ce sens, voir Gautié (2002)(2003) et (2004)). On vise à dépasser le point de vue de l'établissement et même de la firme pour reconstruire la stabilisation des travailleurs dans une dimension de réseau et de réputation. Ces préconisations ne prennent toutefois tout leur sens que dans le contexte mentionné plus haut d'une maîtrise de la conjoncture et d'une « mise sous pression » du marché du travail. Les MTT ne se conçoivent que dans cette optique plus globale d'un meilleur contrôle collectif de l'ensemble des trajectoires de travail rémunéré et non rémunéré. 


\section{Conclusion}

Cette contribution avait pour but de présenter et de discuter l'apport des MTT au débat sur la prise en charge des restructurations. Leur démarche consiste, on l'a vu, à relier les « trajectoires » en cause à l'ensemble des «transitions » qui se produisent sur le marché du travail et autour de lui. Il en résulte un certain nombre de diagnostics et de propositions.

Nous avons tenté de montrer qu'il s'agit d'une voie moyenne ouverte, entre un diagnostic purement local ou spécialisé sur les mérites ou les défauts d'une transition donnée et l'étude des processus de financiarisation, des choix globaux de gestion des firmes et des déterminants macroéconomiques. Ces dimensions échappent en tant que telles au champ des MTT, mais le réseau des interdépendances qu'ils analysent est explicitement lié à ces processus, qu'ils cherchent à influencer.

« Aide toi, le ciel (des choix amont et des macro - politiques) t'aidera », tel pourrait être le slogan auquel conduit cette approche. Si elle propose des perspectives de réponses tant sur le plan curatif, aval en quelque sorte, que sur le plan préventif amont, celles-ci demeurent souvent en pointillés et leur articulation aux autres mesures nécessaires n'est pas suffisamment affermie. Beaucoup reste aussi à faire pour les adapter aux nécessités et opportunités du terrain. Gérer le processus de destruction créatrice mis en évidence par Schumpeter demeure un défi à l'imagination créatrice.

\section{Références :}

Beaujolin, Rachel, (1998), Les vertiges de l'emploi, Grasset

B.I.T. (2004), World Employment Report 2004, Genève

Bolstanski, Luc et Chiapello, Eve (1999), Le nouvel esprit du capitalisme, Gallimard

Burzlaff, Helen, et Le Padellec, Jean-Pierre (2001), La stratégie du mouvement, Paris, Editions Liaisons

Cahuc, Pierre et Zylberberg, André (2004), Le chômage, fatalité ou nécessité ?, Flammarion

Cahuc, Pierre et Kramarz Francis (2004), De la précarité à la mobilité : vers une sécurité sociale professionnelle, Rapport pour les ministres du travail et de la protection sociale

Freyssinet, Jacques (1982), Politiques d'emploi des grands groupes en France, Presses Universitaires de Grenoble

Gautié, Jérôme (2002), « Déstabilisation des marchés internes et gestion des âges sur le marché du travail : quelques pistes », Document de travail du CEE. 
Gautié, Jérôme (2003), «Quelles voies pour l'après-fordisme? Repenser l'articulation entre marché du travail et protection sociale », Esprit, novembre

Gautié, Jérôme (2004), « Les marchés internes, les salaires, l'emploi », Revue Française d'Economie, avril

Gautié Jérôme et Gazier Bernard (2003) "Equipping markets for people : Transitional Labour Markets as the central part of a new social model" Society for the Advancement of SocioEconomics, Conférence 2003 Aix - en - Provence

Gazier, Bernard (2003a), Tous "Sublimes". Vers un nouveau plein-emploi, Paris, Flammarion

Gazier, Bernard (2003b), « Les Marchés Transitionnels du Travail », in Allouche José (dir) (2003), Encyclopédie des Ressources Humaines, Vuibert pp. 862 - 870

Gazier, Bernard (2003c), « L’employabilité », in Allouche José (dir), (2003), Encyclopédie des Ressources Humaines, Vuibert, pp. 418 - 427

Gazier, Bernard et Schmid, Günther (2001), Transitional Labour Markets and their Impact on Industrial Relations, Report to the High Level Group on Industrial Relations and Change, European Commission, Employment and Social Affairs Directorate, août, 22 p.

Giddens Anthony (1998), The Third Way, Polity Press

Raveyre, Marie (2001), "Implication territoriale des groupes et gestion du travail et de l'emploi”, Revue de l'IRES

Schmid, Günther et Gazier, Bernard (dir) (2002), The dynamics of Full Employment. Social Integration through Transitional Labour Markets, Cheltenham, Edward Elgar

Supiot, Alain (dir) (1999), Au-delà de l'emploi, Paris, Flammarion

Winter - Ebmer, Rudolf, (2001) : "Evaluating an innovative redundancy - retraining project : the Austrian Steel Foundation", Discussion Paper n ${ }^{\circ} 277$, IZA, Bonn 